KU-HEP-92-25

\title{
Systematic Analysis Method for Color Transparency Experiments
}

\author{
Pankaj Jain and John P. Ralston \\ Department of Physics and Astronomy \\ The University of Kansas \\ Lawrence, KS-66045-2151
}

\begin{abstract}
We introduce a data analysis procedure for color transparency experiments which is considerably less model dependent than the transparency ratio method. The new method is based on fitting the shape of the A dependence of the nuclear cross section at fixed momentum transfer to determine the effective attenuation cross section for hadrons propagating through the nucleus. The procedure does not require assumptions about the hard scattering rate inside the nuclear medium. Instead, the hard scattering rate is deduced directly from the data. The only theoretical input necessary is in modelling the attenuation due to the nuclear medium, for which we use a simple exponential law. We apply this procedure to the Brookhaven experiment of Carroll et al and find that it clearly shows color transparency: the effective attenuation cross section in events with momentum transfer $Q^{2}$ is approximately $40 \mathrm{mb}\left(2.2 \mathrm{GeV}^{2} / \mathrm{Q}^{2}\right)$. The fit to the data also supports the idea that the hard scattering inside the nuclear medium is closer to perturbative QCD predictions than is the scattering of isolated protons in free space. We also discuss the application of our approach to electroproduction experiments.
\end{abstract}


1. Color transparency $[1,2]$ is a theoretical prediction that under certain circumstances the strong interactions may appear to be effectively reduced in magnitude. Brodsky and Mueller [1] suggested measuring attenuation in a nuclear target as a signal of color transparency. However, to make a quantitative measurement of the attenuation, one must have a value for the hard scattering rate. In the absence of a normalization of the hard sub-process, it is clear that only a combination of the hard scattering rate and attenuation rate is measured in an experiment.

Theory at present cannot supply absolute normalizations for exclusive processes, so the scattering rate in an isolated hadron has been used previously as a benchmark for comparison with the nuclear target. The "transparency ratio" $\mathrm{T}\left(Q^{2}, \mathrm{~A}\right)$ was introduced by Carroll et al [3]: it is the ratio of a cross section measured in the nuclear target to the analogous cross section for isolated hadrons in free space. The transparency ratio is convenient in experimental analysis because some of the uncertainty in the flux and other experimental uncertainties cancel.

However, it has become clear that color transparency experiments cannot be treated on such simple terms. The cross section for isolated $\mathrm{pp} \rightarrow \mathrm{pp}$ scattering shows oscillations which are model- independent evidence for interference among competing amplitudes. In dividing by the isolated (free-space) pp cross section, one creates a certain bias, and indeed possibly a misleading energy dependence in the transparency ratio [4]. On the other hand, if one does not divide by the free space cross section, but simply looks at the $Q^{2}$ and A dependence of the nuclear target, what is the magnitude of the process to be compared to? The experimental data in the nuclear target actually tracks an $\mathrm{s}^{-10}$ naive power law behavior very well [5], but this data represents a combination of the underlying hard scattering and the attenuation. It is a model, and therefore not safe, to assume that the hard process goes like $\mathrm{s}^{-10}$, where $\mathrm{s}$ is the c.m. energy -squared. In fact, the free space data averages about $\mathrm{s}^{-9.7}$, while apparently containing two components, and the whole question of logarithmic effects presents a potentially large correction not totally under theoretical control.

The problem with interpreting the nuclear target data is that an overestimate of the hard scattering rate inside the nucleus might be partially compensated by an underestimate of the nuclear transparency. If one chooses, for example, to "normalize" the nuclear hard scattering rate to $\mathrm{Z}$ times some average of the $\mathrm{pp} \rightarrow \mathrm{pp}$ data, one would be assuming that such hard processes occurred equally often in free space as in the nuclear medium. However, the filtering away of the oscillations indicate this is not true. In fact, the assumption probably overestimates the true rate of "minievents" occurring inside the nucleus. In general, when one studies the ratio of nuclear to free space quantities even at any fixed energy, one is likely to be comparing two unlike processes, and opening the door to a possibly unscientific procedure and confusion.

This problem has gotten the most attention in the hadron-hadron case. However, 
the essential arbitrariness of choosing to divide a nuclear quantity by a free-space, isolated hadron quantity can create uncertainty in all color transparency experiments. Electroproduction is not immune to the problem, because the free space form factors are not totally established as short distance objects, and their interpretation is certainly model dependent. Suppose, for example, that for a certain kinematic point $50 \%$ of the scattering rate in free space were due to processes which do not occur in a nuclear target. When one interpreted the transparency ratio one might then overestimate the scattering rate in the nuclear target by a factor of two. By fitting the data with this assumption, the survival rate due to attenuation would be underestimated by $50 \%$. This simple difficulty is quite a serious issue in electroproduction, because a hadron crosses the target only once. Then, as we show below, a measurement of an attenuation cross section with limited statistics can be completely upset by a change in normalization. However, with good enough statistics one can actually separate the hard scattering rate and attenuation rate in the new way we describe.

2. In this paper we outline a data analysis procedure which is much less model dependent than the transparency ratio method. We never assume the transparency ratio measures attenuation, because the transparency ratio is a ratio between two unknowns. We recommend a global fitting procedure to use all the information in sets of data, with a free parameter setting the normalization of the experiment. A crucial point is that the shape of the cross section with A for a set of nuclear targets contains a great deal of information, and is the best way to deduce the effective attenuation cross section. The normalization of the data is then determined empirically, without excessive model- dependence. The normalization does not have to be set using isolated hadron events, and in fact the empirically determined normalization generally will disagree with the normalization assumed from comparing to such "free space" data. This is progress: we can use the data itself to learn about the differences between the nuclear and free space cases.

We first consider the BNL experiment and its A dependence at fixed beam energy. The experimental data as plotted by Heppelmann [5] is shown in Fig. (1). We analyze the cross section in the nuclear target, dispensing with the transparency ratio. Recall that the events from the nuclear target are taken at the kinematic point corresponding to $90^{\circ} \mathrm{cm}$ scattering; the beam energy $E$ then determines $s=2 m E+2 m^{2}$. We let $Q^{2}$ be the momentum transfer, $Q^{2}=-t=s / 2-2 m^{2}$. So long as the amplitudes have a decent factorization, we can assume that the cross section is a product of a hard scattering probability and an attenuation probability. We find the attenuation from interactions with cross section $\sigma_{\text {eff }}$ for a proton leaving a nucleus via a straight line path. We assume the hard scattering occurs at random over the volume of the nucleus. We then fit the data to a Monte-Carlo event generator 7 in which $\sigma_{\text {eff }}$ is a free parameter, using the parametrizations of the nuclear density given in [6]. The

${ }^{*}$ We thank Steve Hepplemann for lending us the Monte Carlo code used in Ref. (5). 
Monte Carlo propagates the incoming proton to the hard scattering point, and follows the outgoing protons along all kinematically allowed random paths. At each point of each path the loss rate is proportional to the flux, the number density and $\sigma_{\text {eff }}$. For reference we have plotted the nuclear number densities in Fig.(2); there is considerable variation at the edges. It is doubtful that including more detailed structure of the nucleus such as correlations would be justified within the experimental and theoretical errors. Typical results of the Monte-Carlo are shown in Fig.(3), where the dashed lines are smooth fits to the Monte-Carlo output.

Since the Monte-Carlo is a "black-box" a useful consistency check is an analytic representation. The simplest thing is to calculate the survival $P_{p}$ of one proton crossing on a straight line, assuming uniform nuclear density $\rho=(1 / 6) \mathrm{fm}^{-3}$ and nuclear radius $(1.2 \mathrm{fm}) A^{1 / 3}$. An approximate survival probability is then given by cubing the result and renormalizing. The volume-averaged survival probability $P_{p p p}\left(\sigma_{e f f}, A\right)$, which we call the "survival factor", is then given by

$$
\begin{aligned}
P_{p p p}\left(\sigma_{e f f}, A\right) & \cong 1.9\left(\frac{3}{4 n \sigma_{e f f} 1.2 A^{1 / 3}}+\frac{3}{8 n^{3} \sigma_{e f f}^{3} 1.2^{3} A}\right. \\
& \left.\times\left[\exp \left(-2 n \sigma_{e f f} 1.2 A^{1 / 3}\right)-1+2 n \sigma_{e f f} 1.2 A^{1 / 3} \exp \left(-2 n \sigma_{e f f} 1.2 A^{1 / 3}\right)\right]\right)^{3}
\end{aligned}
$$

where $\sigma_{\text {eff }}$ is the attenuation cross section in units of $f \mathrm{~m}^{2}$. With the renormalization factor 1.9 this primitive approximation works surprisingly well for $A \geq 7$ and the analysis of the data agrees within errors. We do not think that few nucleon effects are well accounted for by either method, so we restrict ourselves to $A \geq 7$. For the case of an electroproduction experiment, there is only one proton which must cross the nucleus, and we use a survival factor $P_{p}$ which is $\left(P_{p p p} / 1.9\right)^{1 / 3}$. A main objective of color transparency experiments is to determine the survival factor $P\left(\sigma_{e f f}, A\right)$, or equivalently the attenuation cross section $\sigma_{e f f}$ as a function of energy or momentum transfer.

3. In step (1) of our procedure, for each energy we fit the shape of the nuclear cross section to determine a free normalization $N\left(Q^{2}\right)$ and an attenuation cross section $\sigma_{e f f}\left(Q^{2}\right)$. For the BNL experiment this is:

$$
s^{10} \frac{d \sigma_{A} / d t}{Z}=N\left(Q^{2}\right) P_{p p p}\left(\sigma_{e f f}\left(Q^{2}\right), A\right)
$$

The factors of $s^{10}$ and $1 / \mathrm{Z}$ are simply definitions to take out some typical orders of magnitude - their usage will introduce no bias. Since $\sigma_{e f f}\left(Q^{2}\right)$ and the normalization $\mathrm{N}\left(Q^{2}\right)$ are free, the fit to the data may or may not show that these parameters vary with energy. Of course, finding a variation with energy of $\sigma_{\text {eff }}$ was the goal of color transparency, but at this stage the fitting is an objective, empirical procedure. In 
the BNL data, for example, the best $\chi^{2}$ fit to the shape of the nuclear cross section is obtained by

$$
\begin{aligned}
& \sigma_{e f f}(E=6 \mathrm{GeV})=17_{-3}^{+5} \mathrm{mb} ; \quad N(E=6 \mathrm{GeV})=(5.4 \pm 0.4) \zeta \quad \chi^{2}=0.28 \\
& \sigma_{\text {eff }}(E=10 \mathrm{GeV})=12_{-4}^{+5} \mathrm{mb} ; \quad N(E=10 \mathrm{GeV})=(3.3 \pm 0.4) \zeta \quad \chi^{2}=0.53
\end{aligned}
$$

where $\chi^{2}=\Sigma\left[\left(y_{i}-d_{i}\right) / \Delta d i\right]^{2}, d_{i}$ are the data points, $\Delta d_{i}$ is the error in $d_{i}$ and $y_{i}$ are the theoretical values calculated using the Monte-Carlo, and $\zeta=5.2 \times 10^{7} \mathrm{mb} \mathrm{GeV}^{18}$ is a constant containing the overall normalization of the cross section. Note that $\chi^{2}$ has not yet been divided by the number of degrees of freedom: this is discussed below. The fits for $6 \mathrm{GeV}$ and $10 \mathrm{GeV}$ are shown in Fig. (4a) and Fig. (4b) respectively; the low $\chi^{2}$ values indicate rather good fits to five data points. (The fit does not change significantly if the data point of Lithium is deleted: $\sigma_{e f f}(\mathrm{E}=6 \mathrm{GeV})=\left(19_{-6}^{+17}\right) \mathrm{mb}$ and $\sigma_{e f f}(\mathrm{E}=10 \mathrm{GeV})=\left(13_{-4}^{+7}\right) \mathrm{mb}$ in that case.) At these beam energies we assign the nominal values of $Q^{2}=4.8 \mathrm{GeV}^{2}$ and $8.5 \mathrm{GeV}^{2}$, respectively - this is discussed below. The important thing to notice is the curvature of the plots, which is the main determiner of the value of $\sigma_{e f f}$ - its effect is easily separated from the normalization constant I. To illustrate this point we have also included in Figs.(4a, 4b) the best fits with various permutations of $\sigma_{\text {eff }}$. When $\sigma_{\text {eff }}$ is constrained to the wrong values of 12 $\mathrm{mb}$ and $36 \mathrm{mb}$ at $Q^{2}=4.8 \mathrm{GeV}^{2}$, the $\chi^{2}$ values for the best arbitrary normalization rise by an order of magnitude to 3.1 and 5.3 respectively. Similarly, when $\sigma_{\text {eff }}$ is constrained to the wrong values of $17 \mathrm{mb}$ and $36 \mathrm{mb}$ at $Q^{2}=8.5 \mathrm{GeV}^{2}$, the $\chi^{2}$ values for the best arbitrary normalization rise to 1.6 and 5.3 respectively. Although fixing $\sigma_{\text {eff }}$ reduces the number of parameters, the $\chi^{2}$ per degree of freedom rises to $(0.78$, $1.3,0.4,1.3)$ for the four cases above, from four to thirteen times bigger than the value of 0.093 of our best fits. This means that the best fit is objectively better that the others by a significant statistical measure; the proposal to fit the data with a 36 mb cross section is highly disfavored compared to the best fit.

Having fit the data's A dependence, the fit can be examined at fixed A to find the survival factor as a function of $Q^{2}$ for fixed A. The BNL data was taken only at two energies for a good range of $\mathrm{A}$, so we have $N\left(Q^{2}\right)$ and $\sigma_{e f f}\left(Q^{2}\right)$ at two points. However, each fixed beam energy actually probes a range of true event $\mathrm{cm}$ energies and $Q^{2}$ because of Fermi motion. What can be deduced from an event at a single beam energy depends strongly on how many momenta are measured among the outgoing protons. With enough momentum resolution and variables measured, the Fermi motion is actually measured independently, and this was done well enough at BNL. This procedure produced data at intermediate energies for the Aluminum target. For the other targets, the data was re-binned to increased its statistical significance. This causes a potential "binning problem" because the spread due to Fermi motion

${ }^{\dagger}$ If the data is divided by some arbitrary value, then the shape of the A dependence cannot be fit as well, and $\sigma_{e f f}$ is forced to whatever value is needed to reproduce the normalization of the data 
effect is not exactly symmetric, but inspection of the Aluminum data shows that this should not be a big effect. We therefore accept the data as it is given at the re-binned points.

We can make a function for $N\left(Q^{2}\right)$ which interpolates with $Q^{2}$ smoothly between the two endpoints where $Q^{2}$ Jwas reported. Then inverting (2) we have

$$
\frac{\left(Q^{2} / 4.8 G e V^{2}\right)^{0.86}}{5.4 \zeta} \frac{s^{10}}{Z} \frac{d \sigma_{A}}{d t}=P_{p p p}\left(\sigma_{e f f}\left(Q^{2}\right), A\right)
$$

This is an implicit relation for $\sigma_{e f f}\left(Q^{2}\right)$. We also have the Aluminum data at intermediate values of $Q^{2}$ which can be used to determine $\sigma_{\text {eff }}\left(Q^{2}\right)$. The resulting best consistent fit for $\sigma_{e f f}\left(Q^{2}\right)$ is shown in Fig. (5). We emphasize that future data would be most useful if reported for all $\mathrm{A}$ and all $Q^{2}$ in which case determining $\sigma_{\text {eff }}\left(Q^{2}\right)$ is more direct. With $\sigma_{e f f}\left(Q^{2}\right)$ determined the survival probability itself as a function of $Q^{2}$ is plotted in Fig. (6). The survival factor would be flat with $Q^{2}$ if one used a traditional Glauber model. The fact that the survival probability rises with $Q^{2}$ is clear evidence for discovery of color transparency. It has gone unnoticed in studies involving the transparency ratio, because the assumed normalization in that case skews the analysis.

The transparency ratio, of course, has a certain usefulness, because any experimental uncertainty in the overall normalization drops out. In fact, the overall normalization drops out of our method, also: one does not need to know the normalization to determine $\sigma_{\text {eff }}$. If there is one overall uncertainty in the normalization, it also simply scales out, and does not affect the procedure. Thus we are not asking for absolute cross sections, which are usually a difficult thing. Our method could be applied directly to the transparency ratio if desired - the fitting of the shape of the A-dependence is exactly the same. However, in that case the normalization factor $N\left(Q^{2}\right)$ will reflect the $Q^{2}$ dependence of whatever normalization is chosen.

The effective attenuation cross section $\sigma_{\text {eff }}$ should be a universal quantity which can be compared from experiment to experiment. The theoretical basis for this is factorization between the hard scattering and the nuclear propagation factors. The hard scattering is an abstract object- an integral kernel - which is independent of the target, but when convoluted with different wave functions in different targets produces different effects. An approximate fit to the attenuation cross section from the fit is

$$
\sigma_{e f f}\left(Q^{2}\right)=40 m b\left(2.2 G e V^{2} / Q^{2}\right) \pm 5 m b
$$

for $4.8 \mathrm{GeV}^{2}<Q^{2}<8.5 \mathrm{GeV}^{2}$. The decrease with $Q^{2}$ of the attenuation cross section coincides with the rate predicted theoretically on the basis that the cross section goes like the transverse separations $b_{T}^{2}$ of the participating quarks, and that the region of important $b_{T}^{2}=1 / Q^{2}[4,7]$. However, the scale in the numerator of $2.2 \mathrm{GeV}^{2}$ was not predicted. To put the scale in context, it says that the perturbative QCD ideas are 
beginning to apply for $\mathrm{Q} \geq 1.5 \mathrm{GeV}$. This is another way to present the conclusion that color transparency was actually observed in the BNL experiment.

A final consistency check involves looking at the the normalization factor. After taking out the nuclear attenuation effects, according to the perturbative treatment the $Q^{2}$ dependence of $N\left(Q^{2}\right)$ is due to the hard scattering process. We have found that $N\left(Q^{2}\right)$ decreases relative to $s^{-10}$, meaning that the hard scattering rate in the nuclear target is decreasing faster than the naive quark counting model prediction. In perturbative QCD, however, the quark-counting prediction is modified, due to the running coupling $\alpha_{s}\left(Q^{2} / \Lambda_{q c d}^{2}\right)$ and scaling of distribution amplitudes. The perturbative QCD prediction goes like $\alpha_{s}^{10}$ because there are five gluons in the amplitude:

$$
d \sigma / d t_{p Q C D}=\left(\alpha_{s}\left(Q^{2} / \Lambda_{q c d}^{2}\right)\right)^{10} s^{-10} f(t / s)
$$

It becomes interesting to compare the form including powers of $\alpha_{s}$ with the hard scattering rate in the nuclear target. (The $Q^{2}$ dependence of $\alpha_{s}^{10}$ causes serious disagreement with the data for isolated pp scattering in free space with $\Lambda_{q c d} \approx 100$ $\mathrm{MeV}$.) The reason for consulting the perturbative prediction is to see whether the nuclear target has filtered the events down to something like the shortest distance component. Although (5) appears naive it is perfectly adequate because of the usual ambiguity in the choice of scale. We must generate a range of reasonable theoretical predictions by choosing the $Q^{2}$ of $\alpha_{s}$ in a typical range $(-\mathrm{t} / 2)<Q^{2}<(-1.5 \mathrm{t})$. To improve on this theoretically requires a calculation of next to leading logarithms. The effects of certain leading corrections due to anomalous dimensions fall within the range; for example, the asymptotically large $Q^{2}$ prediction amounts to multiplying (5) by $\left[\log \left(Q^{2} / \Lambda_{q c d}^{2}\right)\right]^{-8 \gamma_{1}}$, where $\gamma_{1}$ is the smallest anomalous dimension [8].

For comparison with the nuclear target we form the ratio of the global $s^{-10} N\left(Q^{2}\right)$ to the pQCD predictions (5) and plot the result as solid lines in Fig. (7). (The asymptotic prediction is also illustrated as a dashed line to show that it falls within the region of scale ambiguity of the pQCD predictions. For comparison we show $\mathrm{s}^{-9.7}$, which is well outside the range of the perturbative predictions.) The solid lines of $s^{-10} N\left(Q^{2}\right) /\left(d \sigma / d t_{p Q C D}\left(Q^{2}\right)\right)$ are quite flat, showing good agreement of the BNL nuclear hard scattering data with QCD. For the Aluminum target we have several data points at several $Q^{2}$ which also fall fairly well within the band of perturbative predictions. These are rather spectacular results, but we emphasize that they should be viewed with caution because the BNL experiment was a pioneering one. If it is confirmed by upcoming higher precision data, they will lead a great deal of strength to the idea that QCD is cleaner after filtering in a nuclear target.

4. The procedure used here to analyze the Brookhaven data can be applied directly to quasi-elastic electroproduction. It is important to approach the analysis steps in the right order. Step (1) is to fit the shape of the A dependence of data with the attenuation cross section $\sigma_{e f f}\left(Q^{2}\right)$ as well as the normalization $N\left(Q^{2}\right)$ treated 
as unknown parameters for each $Q^{2}$. For this step the data can be either in the form of the cross section in the nuclear medium or the color transparency ratio, since the normalization is a separate parameter. Following this procedure for several values of $Q^{2}$ will determine the attenuation cross section $\sigma_{e f f}\left(Q^{2}\right)$ as well as the normalization $N\left(Q^{2}\right)$ as functions of $Q^{2}$. The survival factor $P_{p}\left(\sigma_{\text {eff }}\left(Q^{2}\right), \mathrm{A}\right)$, which is the essential measure of color transparency, is then obtained as a function of $Q^{2}$ and $\mathrm{A}$ by reinserting $\sigma_{\text {eff }}\left(Q^{2}\right)$.

For consistency, quasi-exclusive electroproduction experiments such as the SLACNE18 experiment should see the same attenuation cross sections at the same $Q^{2}$. With the fit to the BNL data we calculate the survival factor $P_{p}\left(\sigma_{e f f}\left(Q^{2}\right), \mathrm{A}\right)$. The results are plotted in Fig. (8) as a function of A and in Fig. (9) as a function of $Q^{2}$. Inspecting the log-scale Fig. (8), one sees almost parallel curves, although the values of $\sigma_{\text {eff }}$ in the plot vary from $36 \mathrm{mb}$ to $12 \mathrm{mb}$. Thus one-particle quasi- exclusive electroproduction has a relatively low discriminating power in determining the attenuation cross section. With limited statistics, an electroproduction experiment can certainly determine a curve in the hard scattering rate-attenuation cross section plane; with good statistics, the curvature of the A dependence can independently fix $\sigma_{\text {eff }}$ and the hard scattering rate.

From $\mathrm{N}\left(Q^{2}\right)$ one can extract an effective form factor for the proton in a nuclear target. The form factors in the nucleus are objects of great intrinsic interest. The comparison with the free space form factors is also extremely interesting. Based on the idea that perhaps not all of the free space form factor is really due to short distance, our expectation is that the form factors extracted from a nuclear target such as iron may be normalized by $50-70 \%$ of the free space ones around $Q^{2}=6$ $\mathrm{GeV}^{2}$. The $\mathrm{pQCD}$ prediction for dependence on momentum transfer is

$$
G_{M}\left(Q^{2}\right)_{p Q C D}=\operatorname{const}\left(\alpha_{s}\left(Q^{2} / \Lambda_{q c d}^{2}\right)\right)^{2} /\left(Q^{4}\right)
$$

Thus, if the pattern we observed in the BNL experiment is repeated, the form factors in the nuclear target should show a dependence on momentum transfer that falls significantly more rapidly than the naive quark-counting rule. The effects of the anomalous dimension are again smaller than the effects of scale ambiguity and can be dropped, assuming one varies the $Q^{2}$ scale of $\alpha_{s}$ over a typical range $\left(-Q_{o}^{2} / 2\right)<$ $Q^{2}<\left(-1.5 Q_{o}^{2}\right)$, where $Q_{o}^{2}$ is the photon momentum transfer. This gives us a band of theoretical predictions. In addition there are higher twist effects, as evidenced by the dipole fit to the free space data $G_{M}\left(Q^{2}\right)_{\text {data }}=$ const. $/\left(Q^{2}+.7 \mathrm{GeV}^{2}\right)^{2}$. Assuming somewhat arbitrarily that this cancels out, in Fig. (10) we plot the product

$$
T\left(Q^{2}, A\right)=\text { const. }\left[\alpha_{s}\left(Q^{2} / \Lambda_{q c d}^{2}\right) / \alpha_{s}\left(1 G e V^{2} / \Lambda_{q c d}^{2}\right)\right]^{4} P_{p}\left(\sigma_{e f f}\left(Q^{2}\right), A\right)
$$

as a function of $Q^{2}$ for various A, and with arbitrary "const". The ratio calculated 
in (7) is a very roughf "transparency ratio" using the guess that the hard scattering rate in a large enough nucleus is perturbative enough to show the running coupling. Then we find that the $Q^{2}$ dependence of (7) is practically flat (Fig. (10)). Since we emphasize data analysis here we do not attempt to estimate the constant, which would represent the percentage of form factor (squared) that would be filtered away by the nuclear medium, and also depends on the denominator of $\alpha_{s}$ in (7). The constants cancel out in ratios for $\mathrm{A}>>1$; our calculation of the ratios varies from $\mathrm{Fe} / \mathrm{C}=0.69, \mathrm{Au} / \mathrm{C}=0.48$ at $Q^{2}=3 \mathrm{GeV}^{2}$ to $\mathrm{Fe} / \mathrm{C}=0.78, \mathrm{Au} / \mathrm{C}=0.60$ at $Q^{2}=6.8 \mathrm{GeV}^{2}$. All of these are estimates based on rough ideas. The transparency ratio does not have the same information as the separate extractions of the hard scattering rate and survival probability, and is not a substitute for the simpler and more systematic method we are presenting.

5. In conclusion, we remark that the experimental determination of color transparency seems to be more subtle than was originally supposed. The basic issue is that exclusive hard scattering contains many unknowns, which has made unraveling the data challenging. However, the original concept of color transparency was to use a nucleus as a test medium to observe and compare scattering with the free space processes. We believe this concept is sound and is beginning to yield results. Our method uses more of the information from the experimental data and we have been able to deduce a great deal. The BNL data strongly indicates an attenuation cross section that decreases with $Q^{2}$ at the same rate as perturbatively predicted. The same data strongly indicates a normalization of the hard scattering rate that decreases faster than the free space rate, and is in rather good agreement with perturbative theory. These two results are not totally surprising. Indeed, if we had much faith in the perturbative prediction's dependence on $Q^{2}$ due to many powers of $\alpha_{s}$, then the interplay of a falling cross section and rising survival might have been obvious in the first look at the data. The goal is to have sufficiently strong data and systematic data analysis so that no faith is required.

With the current data everything indicates that the new method is a powerful one which can be productively applied to color transparency experiments in the future. We eagerly await new data, in the hopes that it will confirm our conclusion that color transparency was actually observed by Carroll et.al.

Acknowledgements: This work has been supported in part by the DOE Grant No. DE-FG02- 85-ER-40214.A008. We thank Stan Brodsky, Alan Carroll, Steve Heppelmann, Byron Jennings, and Bob McKeown for useful comments.

\footnotetext{
${ }^{\ddagger}$ In general the transparency ratio depends on the specific cross sections used for comparison and there is no universal agreement or convention established.
} 


\section{Figure Captions}

Fig.(1) The A dependence of the data of Carroll et al. as reported by Heppelmann (Ref. (5)). There is a clear difference in curvature of the A dependence between 6 $\mathrm{GeV}$ and $10 \mathrm{GeV}$ indicating a smaller attenuation cross section at $10 \mathrm{GeV}$. Due to oscillations in the denominator, the relative normalization of the transparency ratio at the two energies is a separate issue.

Fig. (2) Plot of nuclear number densities $\rho$ (r) from Ref.(7) used in the Monte Carlo calculation. The elements $\mathrm{Li}, \mathrm{C}, \mathrm{Al}, \mathrm{Cu}$, and $\mathrm{Pb}$ correspond to nuclear $\mathrm{A}=7,12$, 27, 64, and 207.

Fig. (3) Smooth fits (dashed lines) to the Monte-Carlo calculation (solid lines) of the survival probability $P_{p p p}\left(\sigma_{e f f}, \mathrm{~A}\right)$ as a function of $\sigma_{\text {eff }}$ for various A.

Fig. (4) Fitting the A dependence of the nuclear cross section with attenuation cross sections $\sigma_{\text {eff }}=12,17$, and $36 \mathrm{mb}$ at fixed momentum transfer. (a) $Q^{2}=4.8 \mathrm{GeV}^{2}$. (b) $Q^{2}=8.5 \mathrm{GeV}^{2}$. The solid line shows the best fit. For comparison the dashed lines show constrained fits using permutations of $\sigma_{\text {eff }}$ and best normalizations.

Fig. (5) The attenuation cross section $\sigma_{e f f}$ extracted from the BNL data. Solid line: best fit including the intermediate $Q^{2}$ data points reported for Aluminum. Dashed line: a $36 \mathrm{mb}$ strong interaction cross section for comparison. The two data points are the global $\sigma_{\text {eff }}$ values extracted from Fig.(4); the uncertainty corresponds to the standard definition, producing a change of the fit by one unit of $\chi^{2}$.

Fig. (6) The survival probability $P_{p p p}$ extracted from the BNL data as a function of $Q^{2}$ for various $\mathrm{A}$. The increase with $Q^{2}$ is evidence for observation of color transparency.

Fig. (7) Comparison of the fit to the hard scattering rate $\mathrm{N}\left(Q^{2}\right)$ to pQCD prediction, as given in Eq. (5), including scale ambiguity. The ratio $\mathrm{s}^{-10} \mathrm{~N}\left(Q^{2}\right) /\left(\mathrm{d} \sigma / \mathrm{dt}_{p Q C D}\right)$ is plotted as solid lines; a flat curve indicates agreement. The lower line uses the scale of the running coupling as $\left(Q^{2}=0.5(-\mathrm{t})\right)$ in calculating $\mathrm{d} \sigma / \mathrm{dt}_{p Q C D}$. The upper line uses $\alpha_{s}\left(Q^{2}=1.5(-\mathrm{t})\right)$. Data points are for the complete data set (box symbol) and the Aluminum data (no symbol). The long dashed curve represents $\mathrm{s}^{-9.7} /\left(d \sigma / d t_{p Q C D}\right)$ and the short dashed curve is the asymptotic prediction [8] for $d \sigma / d t_{p Q C D}$ using $\alpha_{s}\left(Q^{2}=-t\right)$ and divided by the right hand side of Eq. 5 .

Fig. (8) Prediction of the survival probability $P_{p}$ in quasi-exclusive electroproduction versus A using $\sigma_{e f f}\left(Q^{2}\right)$ extracted from the BNL data. Note that a change in normalization can nearly compensate a change in $\sigma_{e f f}$.

Fig. (9) Prediction of the survival probability $P_{p}$ in quasi-exclusive electroproduction versus $Q^{2}$ using $\sigma_{\text {eff }}\left(Q^{2}\right)$ extracted from the BNL data. $\mathrm{A}=12,56,197$ for elements $\mathrm{C}, \mathrm{Fe}, \mathrm{Au}$.

Fig. (10) $Q^{2}$ dependence of a "transparency ratio" consisting of $\alpha_{s}^{4}\left(Q^{2}\right) P_{p}$ for Fe (Eq. 7) under the assumption that the nuclear form factor goes like $\alpha_{s}^{2}$. Lower and upper lines show the range of theory predictions from varying the choice of scale $Q^{2}$ of $\alpha_{s}$ from $\left(-0.5 Q_{o}^{2}\right)<Q^{2}<\left(-1.5 Q_{o}^{2}\right)$, where $Q_{o}^{2}$ is the photon momentum transfer. Vertical scale depends on overall normalization of hard scattering rate in nuclear medium which has not been specified. 


\section{References}

1. S. J. Brodsky and A. H. Mueller, Phys. Lett. B 206, 685 (1988), and references therein.

2. S. J. Brodsky and G. F. de Teramond, Phys. Rev. Lett. 60, 1924 (1988); N. N. Nikolaev and B. G. Zakharov, Z. Phys. C49, 607 (1991); J. Botts, Phys. Rev. D 44, 2768(1991); J. P. Ralston and B. Pire, Nucl. Phys. A 532, 155c (1991) and Phys. Lett. B256, 523 (1991); H. Borel, S. Fleck, J. Marroncle, F. Staley, and C.Vallet, Nucl. Phys. A 532, 291c (1991); C. E. Carlson and J. Milana, Phys. Rev. D 44, 1377 (1991); J. P. Ralston, Phys. Lett. B 269, 439 (1991); P. Jain, J. Schechter and H. Weigel, Phys. Rev. D 45, 1470 (1992); O. Benhar, A. Fabrocini, S. Fantoni, V. R. Pandharipande, and I. Sick, Nucl. Phys, A 532, 277c(1991); J. Hufner and M. Simbel, Phys. Lett. B 258, 465 (1991); B. Z. Kopeliovich and B. G. Zakharov, Phys. Lett. B 264, 434 (1991); Phys Rev D 44, 3466 (1991); J. M. Eisenberg and G. Kalbermann, preprint \# PRINT-920126 TEL-AVIV); H. Heiselberg, G. Baym, B. Blaettel, L. L. Frankfurt and M. Strikman, Phys. Rev. Lett. 67, 2946 (1991); T.-S. H. Lee and G. A. Miller, Phys. Rev. C 45, 1863 (1992); G. R. Farrar, H. Liu, L. Frankfurt and M. Strikman, Phys. Rev. Lett. 61, 686 (1988); B. Jennings and G. Miller, Phys. Lett. B236, 209 (1990); Phys. Rev. D 44, 692 (1991); Phys. Lett. B274, 442 (1992); S. Gardner, CEBAF preprint \# CEBAF-PR-92-002.

3. A. S. Carroll et al., Phys. Rev. Lett. 61, 1698 (1988).

4. J. P. Ralston and B. Pire, Phys. Rev. Lett. 61, 1823 (1988).

5. S. Heppelmann, Nucl. Phys. B (Proc. Suppl.) 12, 159 (1990).

6. H. De Vries, et al, Atomic Data and Nuclear Data Tables, 36, 495 (1987).

7. S. Nussinov, Phys. Rev. Lett 34, 1286 (1975); F. E. Low , Phys. Rev. D12, 163, (1975); J. Gunion and D. Soper, Phys. Rev. D15, 2617, (1977).

8. S. J. Brodsky and G. P. Lepage, Phys. Rev. D22, 2157 (1980). 